\title{
Inconduite scientifique? Quelle inconduite?
}

$\mathrm{P}$ ourquoi le Canada a-t-il tant de retard sur ses homologues occidentaux dans l'établissement de mécanismes et processus intégrés pour faire face à l'inconduite scientifique? Est-ce un problème d'inertie? De conflits entre niveaux de compétence? D'une croyance naïve en l'incorruptibilité de tous les chercheurs canadiens? Du concept erroné selon lequel le secret est dans le meilleur intérêt de tous les intéressés parce qu'il protège des salissures la réputation des établissements et des particuliers?

Aucune des raisons ci-dessus ne justifie l'inaction qui perdure, particulièrement à une époque où les résultats scientifiques sont largement diffusés et où les Canadiens les utilisent pour prendre des décisions sur les soins de santé. À juste titre ou non, chaque cas d'inconduite nous salit tous et mine la confiance du public, comme dans le cas des chercheurs canadiens Roger Poisson ${ }^{1}$ et Ranjit Chandra ${ }^{2}$. Si on ne détecte pas la fraude ou que l'on ne redresse pas les dossiers scientifiques, d'autres chercheurs gaspilleront énormément de temps, d'énergie et d'argent à œuvrer dans des impasses ${ }^{3}$. Les décisions sur les soins de santé fondées sur des recherches frauduleuses peuvent en outre mettre en danger la vie et la santé des patients ${ }^{1}$.

Il y a toutefois encore pire, soit l'omission d'agir avec rapidité, fermeté et transparence pour régler les cas d'inconduite. Personne ne peut faire confiance aux résultats de la recherche canadienne si nous cachons les abus. Reconnaissant que la solution fondée sur des principes consiste à établir des politiques et des procédures structurées et concrètes pour réduire au minimum les abus de la recherche et y faire face, d'autres pays ont dépassé le Canada depuis longtemps. Les États-Unis ont créé le Bureau de l'intégrité de la recherche en 1993. En I992, le Danemark a mis sur pied les Comités sur la malhonnêteté scientifique coprésidés par un juge de la cour supérieure. L'Allemagne, la France, la Finlande, l'Australie et la Suède sont au nombre des autres pays qui ont établi de tels organismes. Le Royaume-Uni est en train de mettre sur pied une agence nationale.

Que les fonctions de ces organismes soient de faire enquête ou de conseiller, ils établissent au moins des lignes directrices uniformes et des mécanismes de déclaration, ainsi qu'un mode de suivi et de déclaration des plaintes pour inconduite. Aux États-Unis, le nombre de plaintes révèle une tendance inquiétante dans les milieux de la recherche, qui consiste à pousser l'intégrité à la limite, que ce soit par pure ambition, à cause de la rivalité suscitée par les subventions ou de pressions exercées par des bailleurs de fonds de l'extérieur. Le Bureau de l'intégrité de la recherche des États-Unis a signalé que le nombre de plaintes a atteint 265 en 2005 , soit presque $50 \%$ de plus qu'en 2003. Par contre, l'information canadienne est rare, même si les Instituts de recherche en santé du Canada déclarent avoir reçu 54 plaintes entre 2000 et 2006 . Dans 36 de ces cas, on a jugé qu'il valait la peine de faire enquête (menée par l'université où se serait produite la prétendue inconduite) : dans 2 I cas, on a constaté qu'il y avait eu $\exists$ violation des lignes directrices d'application volontaire du conseil et l'enquête se poursuit dans 3 cas.

Le système canadien de traitement de l'inconduite est si désordonné et opaque qu'il est impossible d'en évaluer l'efficacité avec précision, et encore moins de connaître l'impartialité des enquêtes ou la pertinence des sanctions ou des mesures de redressement, ce qui est tout aussi problématique.
Il faut mettre fin à tout cela. Il faudrait présenter et adopter une législation habilitante afin de créer une agence nationale de l'intégrité de la recherche indépendante et autonome ${ }^{2}$. La structure précise de l'agence, sa composition et ses pouvoirs peuvent faire l'objet de débats, mais le JAMC croit que le nouvel organisme devrait rendre compte au Parlement de l'établissement et de l'application de règles et de procédures nationales de traitement des cas d'inconduite reliée à la proposition, à l'exécution, à l'examen de la recherche et à la production de rapports à ce sujet. L'agence devrait avoir un rôle d'éducation à jouer et être un organisme quasijudiciaire, un peu comme nos ordres provinciaux, peut-être, qui aurait compétence sur tout ceux qui détiennent des postes universitaires et effectuent de la recherche, qu'elle soit financée par le secteur public ou par le secteur privé. L'organisme devrait avoir un service d'enquête - complet et doté de conseillers juridiques afin d'assurer l'application de la loi — pour traiter des cas d'inconduite comme la fraude, la falsification, les conflits d'intérêts et l'impropriété financière, afin d'éviter que les universités et les établissements de recherche aient à enquêter sur leurs propres cas, sauf pour infractions mineures comme les rapports de recherche rédigés par un rédacteur anonyme, la publication en double ou les affirmations trompeuses dans un curriculum vitæ.

Le nouvel organisme devrait avoir à la fois le pouvoir d'appliquer des pénalités appropriées comme le retrait de subventions ou l'interdiction de demander de nouvelles subventions, et la latitude de soumettre des affaires aux services policiers lorsque des accusations criminelles sont justifiées ${ }^{2}$. Il devrait avoir des mécanismes clairs de présentation des plaintes, faire fonction de dépôt national des rapports d'enquête et des statistiques sur l'inconduite, et devrait produire un rapport annuel au public afin que nous puissions garder confiance pendant qu'on dépense des milliards de dollars du contribuable en recherches sur la santé.

N'attendons pas le prochain scandale. Il y en a eu bien assez pour justifier de nous sortir la tête du sable. Les $99 \%$ de chercheurs canadiens exceptionnels qui respectent les principes de l'intégrité, de l'honnêteté et de la confiance méritent une meilleure protection contre les quelques-uns qui sont prêts à abuser de notre confiance.

\section{Wayne Kondro}

Rédacteur aux actualités

Paul C. Hébert

Rédacteur en chef du JAMC

pour l'Équipe de rédaction de l'éditorial du JAMC

(Paul C. Hébert, Amir Attaran, Noni MacDonald et Ken Flegel)

Ottawa (Ont.)

\section{RÉFÉRENCES}

I. Angell M, Kassirer JP. Setting the record straight in the breast-cancer trials. N Engl JMed r994;330:1448-50.

2. Kondro W. Call for arm's-length national research integrity agency. JAMC 2007;176(6):749-50.

3. Council of Science Editors. CSE's white paper on promoting integrity in scientific journal publications. Disponible : www.councilscienceeditors.org/editorial_policies /white_paper.cfm (consulté le ier mars 2007). 